\title{
The application of XML in Network Education
}

\author{
Yang Zhan \\ Jilin Agricultural University, Changchun, China \\ zy9113@163.com
}

\begin{abstract}
HTML language was facing great challenge with the development of network education. XML technology provided a new development and application platform for network education, with its easy to use, flexible, powerful features. It could be widely used in the standardization of resources, multimedia teaching, personalized learning and other aspects.
\end{abstract}

Keywords: XML; Network education; Multimedia; Personalized learning.

\section{Introduction}

XML is a new technology in recent years. It has permeated all fields of the network. XML provides a new platform for the development and application of network education. Network education is a new form of education which is based on the modern information technology platform, which is composed of digital technology, network communication technology, computer multimedia technology and so on. The teaching resources construction is the core of network education. But because there is no uniform standard and technical means, the different systems use different file formats, developed their own independent teaching resources system, system platform, database, data structure, type definition. Then the resource description exist great differences, this caused the online resources are scattered and not standardized. In this states, although there are a lot of teaching resources on the Internet, but resource file format inconsistent, unable to form a unified format, this makes it difficult to share resource content, so it has been the phenomenon of repeated development and waste of resources.

At present, most of the network courseware was based on the HTML document. But it had encountered some problems in the development of network education because of the characteristics of HTML. Its scalability was poor, it could only use the tags provided in the HTML and could not be used for others. HTML just provides the function of displaying the page data, it could not did anything about the description of the content and structure of the document. For example, it using <font> to display fonts and using <bgcolor> to define the background color, etc. The network multimedia resources based on HTML have the disadvantage of being unable to obtain information. The emergence of extensible markup language XML provides an effective solution for the standardization of network education resources and multimedia teaching.

\section{The characteristics of XML}

W3C Recommendation XML as a data representation and exchange on the Web standard in February 1998. It is an optimized subset of SGML, it has become the standard format for data representation and data exchange on the Internet by its own unique advantages [1]. XML is a set of rules for define the semantic tag, these tags divide the document into many parts and represent them. XML tags describe the structure and meaning of the document content, rather than describing the formatting of the page elements. The document itself only described what the document contains, it does not explain what the document looks like.

XML used DTD and XML schema [2] to achieve the schema definition: DTD (document type definition) described what tags can be used in the XML document and the properties of tags; the XML schema was an XML document, It had more data types than DTD, it had better expansibility, scalability and reusability.

XML mainly had the following good characteristics. First is scalability. XML allows users to create and use their own tags rather than the limited vocabulary for HTML. This is very important, 
because we can use XML to define their own tags language for a variety of resource applications, XML was the basis for resource sharing and data exchange. Second, it has the characteristic of flexibility. HTML was difficult to develop further. Because it was the mixture of formatting, hypertext, and graphical user interface semantics and it difficult to develop these features together. XML provides a structured representation of the data, which allows the user to separate the data from the structured data. So, Web users are pursuing a number of advanced features in the XML environment was easy to achieve.Third, it was self descriptive. XML documents usually contain a document type declaration DTD, so the XML document was self described. Not only people can read XML documents, the computer too. XML means that the data was truly independent of the application system, and the data can be reused. XML documents were regarded as the database of the document and the data of the document. In addition, XML also has simplicity. Its complexity was only $20 \%$ of SGML, but it was about $80 \%$ function of SGML. XML was much simpler than the full SGML, easy to learn, easy to use and easy to implement. XML has also absorbed the experience of using HTML on Web for many years. XML has become an open standard for data representation all about these, which was independent of machine platforms, vendors, and programming languages. So it will bring new opportunities for online education.

\section{The application of XML in network education}

\subsection{XML applied to the standardization of educational resources.}

In the past, all kinds of network education resources were distributed on different database platforms, the description of resources have great differences, because of we did not established a unified resource description method.XML can help people to establish such a unified standard. Such as if we want to describe a course name, speaker teachers, course content and so on, which must created each tag, and DTD or XML described the new tag in the document type definition. For example, using a DTD to describe the network courseware:

$<$ ? $\mathrm{xml}$ version $=" 1.0 "$ standalone $="$ no" ?>

$<$ ! DOCTYPE resources [

$<$ ! ELEMENT resources (name, teacher+, detail)>

$<$ ! ELEMENT teacher (teaname) $>$

$<$ ! ELEMENT name (\#PCDATA) >

$<$ ! ELEMENT teaname (\#PCDATA) $>$

$<$ ! ELEMENT detail (\#PCDATA) $>$ ]

In above DTD file, the first line is an XML declaration. Below is a description of the type of document: ELEMENT is used to represent element; \#PCDATA represent data; the teacher is composite elements, "teacher+" indicate teacher can contain one or more. The feature of XML is that multiple documents can be shared commonly by DTD. These DTD can be written by different people. Of course, you can also use XML to describe the structure.

At present, the teaching resources of network education were distributed on multiple servers. The server may be distributed in different regions, each server and the database platform may be different, there are great differences in the information structure, storage organization, retrieval method, query constraint condition and so on. Using XML to provide standard educational resource description method, it can solve the problems of the standardization of information extraction, inquiry, interaction and integration of teaching resources [3].

\subsection{The application of XML in the distance multimedia teaching.}

In the past, most of the remote multimedia teaching methods were HTML, ASP, JSP, PHP and other technologies. It can play the role of Distance Multimedia Teaching by the combination of XML and traditional technology.

XML was used to store data. They all support this format whether it was in the Unix platform, or in the Macintosh platform or Windows platform. If JAVA was a cross platform programming language, then XML was a cross platform data format. As a result of this, they are combined so closely, and 
most of the current XML applications are based on JAVA technology. But many other languages, including Perl, C\#, and other XML also have a very good support. XML was not responsible for running the program and the performance of the form of data. Data expression can be achieved through the XSL or CSS. XML can also be used to a small database, many large and medium-sized databases also had a very good support for XML such as Oracle, SQLServer, and MySQL [4]. People can use XML technology to construct the system structure of a remote multimedia courseware system.

The structure of this kind of Long-distance Multimedia courseware system was mainly divided into customer service layer, application service layer and database service layer:

The first layer was the customer service layer. It was the interface between the user and the whole system, it providing the user interface. For teachers, it can be realized on the courseware of the relevant information to add, modify, delete and other operations; for distance learners, it can be through the browser for online learning, online information query, etc.

The second layer was the application service layer. It includes Internet information server, it can start the process to respond to customer requests and support the function of XML update, and it can transfer the data changes on the database server to the user. When receiving the XML document from the client, explains this document by using XML parser, and then access the database server according to its requirements. Finally, combination of these information, and dynamically generate a corresponding requirements of the XML file, sent it to the client.

The third layer was the database service layer. It was mainly composed of XML files and relational database. It stores information about the contents of the network courseware and display styles, etc. It applies ODBC and SQL to connect with application service layer, it provides data query and storage and other functions.

This structure not only can improve the efficiency of the release of the foreground, but also improve the retrieval efficiency of the background database, so it improves the performance of the computer server. Application of XML technology can make the distance teaching more vivid, the learners can better understand the content of teaching. In the same, the XML can also be used in the distance learning platform to establish a XML based online examination system and online question answering system. The combination of XML technology and multimedia technology has overcome the limitation of the traditional distance education, and it was helpful to form an active, cooperative and open learning mode.

\subsection{The application of XML in personalized learning environment.}

Personalized learning environment is an important place for "after-school learners" or "irregular learners", we using XML to achieve this environment can display personalized learning content more quickly and easily.

At present in the process of online learning, the learners and remote network server needs to be kept for a long time "connection. The learner send out the request to the server, server return the results in the form of HTML to the learners, but one web server can handle only a limited connection at the same time. XML introduces new web server software, HOTMETAL server software, which can add a new "middle tier" server to the whole network system. It constitutes a three layer web structure. In the three layer web model, the middle tier software can obtain information from the remote database to meet the needs of customers, this information can be XML files, you can also through ODBC or other similar database connection way to obtain information. Once the middle tier layer software assembled the information together, it can use the form of XML to the user.

The nature of the middle tier of the WWW is merge the data, when the learners logged on system, the system gathered the remote data and packaged it into a XML document according to the learning progress or the requirements of learners, and then sent it to the learner. In this way, the learner didn't need to continue connect with the server. And it can make full use of the distance teaching database to realize the personalized learning environment. 


\section{Summary}

Although there are some unsolved problems in XML, but its great superiority has been revealed. XML has a broad application prospects in the field of network education, it will bring fundamental innovation in many aspects of network education.

\section{Reference}

[1] Information on: www.w3.org

[2] Didier Martin. Professional XM1. Wrox press, 2001, p.5-7.

[3] Wenying Pang. The application of XML technology in Distance Education. Journal of Hainan Radio \& TV University.2007 (2), p.72-74.

[4] Information on: www.xml.org.cn

[5] Zhongyang Wang, Yi Ji. Design of the XML-based Resource Description and Management System in Distance Education. Computer Application. Vol. 24 (2004) No.6, p171-173. 